\title{
High-resolution MEMRI characterizes laminar specific ascending and descending spinal cord pathways in rats
}

\section{Vijai Krishnan ${ }^{1,2,3,4}$, Jiadi Xu ${ }^{4}$, German Alberto Mendoza ${ }^{4}$, Alan Koretsky ${ }^{5}$,} Stasia A Anderson ${ }^{6}$, and Galit Pelled ${ }^{1,2,3,4} *$

${ }^{1}$ Department of Biomedical Engineering, Michigan State University, East Lansing, MI, United States

${ }^{2}$ The Institute for Quantitative Health Science and Engineering, Michigan State University, East Lansing, MI, United States

${ }^{3}$ Department of Radiology, Michigan State University, East Lansing, MI, United States

${ }^{4}$ Johns Hopkins Medicine Department of Radiology and Radiological Science, Baltimore, MD, United States

${ }^{5}$ Laboratory of Functional and Molecular Imaging, National Institute of Neurological Disorders and Stroke, National Institutes of Health, Bethesda, MD, United States

${ }^{6}$ National Heart, Lung, and Blood Institute, National Institutes of Health, Bethesda, MD, United States

* Corresponding author

Keywords: Manganese enhanced magnetic resonance imaging, Spinal cord, Motor neurons, Laminae 


\begin{abstract}
Manganese Enhanced MRI (MEMRI) utilizing different manganese chloride $\left(\mathrm{MnCl}_{2}\right)$ delivery methods, has yielded valuable architectural, functional and connection information about the brain. MEMRI also has the potential in characterizing neural pathways in the spinal cord. The spinal cord grey matter is anatomically composed of nine distinct cellular laminae, where each of the laminae receives input from a specific type of neuronal population and process or serves as a relay region in a specific sensory or motor pathway. This type of laminar arrangement in the spinal cord is currently only visualized by histological methods. It is of significant interest to determine whether laminar specific enhancement by $\mathrm{Mn}^{2+}$ can be achieved in the spinal cord, as has been reported in the brain and olfactory pathway. Here we focus on using MEMRI to determine the specific laminae of the thoracic region of the spinal cord. We focus on $\mathrm{MnCl}_{2}$ changes in the ascending and descending tracts of the spinal cord. Major factors in applying this technique in the spinal cord are the ability to acquire high-resolution spinal cord images and to determine a noninvasive route of administration which will result in uptake by the central nervous system.
\end{abstract}

We have applied the MEMRI approach by intraperitoneal (i.p). delivery of $\mathrm{MnCl}_{2}$ and imaged lumbar and thoracic spinal cord levels in rats to determine whether $\mathrm{T}_{1}$ weighted MRI can detect spinal cord laminae 48 hours following $\mathrm{MnCl}_{2}$ administration. $\mathrm{T}_{1}$ weighted images of the lower lumbar level were obtained from $\mathrm{MnCl}_{2}$ injected and control rats. Here we demonstrate laminar specific signal enhancement in the spinal cord of rats administered with $\mathrm{MnCl}_{2}$ vs. controls in MRI of the cord with ultra-high, $69 \mu \mathrm{m}$ in-plane resolution. We also report reduced $\mathrm{T}_{1}$ values over time in $\mathrm{MnCl}_{2}$ groups across laminae I-IX. The regions with the largest $\mathrm{T}_{1}$ enhancements were observed to correspond to laminae that contain either high cell density or large motor 
neurons, making MEMRI an excellent tool for studying spinal cord architecture, physiology and function in different animal models.

\section{Introduction}

The spinal cord consists of nine laminae that have different cytological and functional characteristics. The large somatic motor neurons that occupy laminae IX are essential for maintaining muscle tone and innervating muscle spindles (1). Neuronal death in that region can lead to a number of motor neurons disorders, such as amyotrophic lateral sclerosis (ALS) (2, 3), spinal muscular atrophy, spinal bulbar muscular atrophy and spinal muscular atrophy with respiratory distress 1 (4). To date, motor neuron diseases are untreatable and may be fatal, as in ALS, or cause severe clinical symptoms. Most of the motor neuron diseases initially affect only a specific cell population within the spinal cord and does not result in gross architectural changes. Using non-invasive imaging tools to monitor the viability of the large motor neurons in the spinal cord could greatly contribute to the ongoing efforts of finding new therapeutic strategies for motor neuron disorders. There is an ongoing need to develop technologies that will allow non-invasive imaging of spinal cord function. Nevertheless, in vivo highresolution imaging of the spinal cord remains a challenge due to several factors such as the susceptibility to motion, variation in tissue depth over the length of the cord, and artifacts from the cerebrospinal fluid (CSF) and vertebrae.

Manganese enhanced magnetic resonance imaging (MEMRI) has been applied to identify and trace connectivity changes in specific neuronal architecture of the brain and spinal cord. MEMRI has also used to assess functional information pertaining to different areas of the brain affected by a variety of brain diseases (5-8). The $\mathrm{Mn}^{2+}$ uptake by 
neurons has been shown to be tightly coupled to the neuronal activity (9-14), and hence, the cells viability. $\mathrm{Mn}^{2+}$ ions have been shown to accumulate in the desired tissue leading to a subsequent shortening of $\mathrm{T}_{1}$ weighted intensity. This in turn leads to a favorable contrast enhancement in $\mathrm{T}_{1}$-weighted MRI signaling in the tissue. Therefore, changes in $\mathrm{T}_{1}$-weighted signal values of different spinal cord regions after $\mathrm{Mn}^{2+}$ delivery may provide an indication of the cells function. Indeed, it has been demonstrated that MEMRI can detect lesions and $\mathrm{Mn}^{2+}$ transport within spinal cord neurons can give an indication of the degree of the spinal cord recovery in rodents (15-17).

Here we demonstrate that $48 \mathrm{~h}$ following systemic injections of $\mathrm{MnCl}_{2}$ in rats, there is a significant accumulation of $\mathrm{Mn}^{2+}$ ions within localized spinal cord laminae, specifically in laminae that consist of large motor neurons, as well as in laminae that consist of particular high cell density. We also report that MEMRI enhanced visualization of laminae regions I-IX of the thoracic segment of the rat spinal cord. These findings were further correlated with immunostaining. These findings can be used to assess discrete laminar specific changes of the spinal cord in vivo.

\section{Methods}

$\mathbf{M n C l}_{\mathbf{2}}$ administration: Animal procedures were performed in accordance with Johns Hopkins animal care and use committee guidelines. Male Sprague-Dawley rats (200g) were anesthetized with $2 \%$ Isoflurane and $2 \mathrm{ml}$ of $100 \mathrm{mM} \mathrm{MnCl} 2$ dissolved in saline was delivered i.p. with $0.4 \mathrm{mmol} / \mathrm{kg}$ of body weight $(\mathrm{n}=8)$. Control rats received only saline injections $(n=3)$. 
Animal preparation for MR: 48 hours following $\mathrm{MnCl}_{2}$ administration, rats were anesthetized with $2 \%$ Isoflurane and were placed supine (in order to suppress motion) in a secured MR head and body cradle. A $4 \times 1$ phased receiving coil was placed under the rats thoracolumbar vertebrae. The head was slightly stretched and tilted back to reduce curvature at the cervical region. The head, body, and legs were secured with tape during the experiment and the breathing rate was monitored. Each rat was imaged before and after $\mathrm{MnCl}_{2}$ administration.

MRI: An ultra-high field 11.7 Tesla/16 cm horizontal bore small-animal scanner (Bruker BioSpin, Rheinstetten, Germany) was used for imaging. A $72 \mathrm{~mm}$ quadrature volume resonator was used as a transmitter. $T_{1}$ weighted images were collected using rapid acquisition with refocused echoes (RARE) imaging module with an echo time of 14 ms, RARE sequence with 2 echoes, slice thickness $=1 \mathrm{~mm}$, a matrix size of $320 \times 320$ and a field-of-view (FOV) of $2.2 \times 2.2 \mathrm{~cm} . \mathrm{T}_{1}$ maps were measured using a RAREVTR sequence (RARE with six variable repetition times 500, 700, 1000, 1500, 2000, 5000 ms). The matrix size and FOV were identical to the $\mathrm{T}_{1}$ weighted images. 17 slices were acquired, beginning at the lower lumbar area and moving up towards the cervical region. Three saturation slices around the spinal cord area were used to allow for a reduced FOV around the cord without alias. A fat suppression module was utilized in all experiments.

Histology: At the end of the imaging session, rats were perfused with $200 \mathrm{ml}$ of saline and then $200 \mathrm{ml}$ of $4 \%$ paraformaldehyde in $0.1 \mathrm{M}$ phosphate buffer (PBS), pH 7.4. The lumbar area of the spinal cord was removed and post-fixed in perfusion solution for $2 \mathrm{~h}$ at $4^{\circ} \mathrm{C}$ and cryoprotected in $30 \%$ sucrose solution in PBS. Sectioning was done with a freezing microtome (50 $\mu \mathrm{m}$ thick slices) and sections were mounted onto slides. Sections 
were thawed to room temperature and blocking $(3 \times 10$ min) was done with PBSG-T $(0.1 \mathrm{M}$ PB pH $7.4+0.9 \% \mathrm{NaCl}+0.2 \%$ gelatin $+0.2 \%$ triton $)$. Neuronal nuclei were incubated with NeuN monoclonal antibody (Chemicon) at a 1:1000 dilution for $2 \mathrm{~h}$ at room temperature. Primary antibody was incubated overnight at $4^{\circ} \mathrm{C}$ and then washed 3 $\times 20 \mathrm{~min}$ in $\mathrm{PBS}, \mathrm{pH}$ 7.2. Immunoreactivity was visualized with the appropriate secondary antibody conjugated to Alexa 488 (1:1000 dilution; Molecular Probes, Eugene, OR). Sections were counterstained with DAPI, which labels all cell nuclei. Sections were rinsed (3x10 $\mathrm{min})$ in PBSG-T and incubated with an anti-mouse biotinylated secondary antibody (1:500, Chemicon) for 45 minutes at room temperature. For DAB detection, sections were rinsed $(3 \times 10 \mathrm{~min})$ in PBSG-T and incubated with Strepavidin HRP diluted in Tris buffered saline (1:500, Chemicon) for 30 minutes at room temperature. Sections were rinsed $(2 \times 10 \mathrm{~min})$ with phosphate buffered saline $+0.2 \%$ triton, and $(2 \times 10 \mathrm{~min})$ with phosphate buffered saline alone. Detection was performed with DAB chromagen reagent (Chemicon) incubated for 1-5 minutes and rinsed with PBS when staining was optimal. All images were acquired at $20 \times$ magnification using a computer-controlled microscope (Zeiss Axio Observer Z.1).

Data Analysis: The mean $\mathrm{T}_{1}$ values and standard error was calculated for each region-of-interest (ROI). $\mathrm{T}_{1}$ values were calculated using Matlab. $\mathrm{T}_{1}$ values are represented as mean \pm SEM. Data was analyzed by paired t-test and results were considered significant if $\mathrm{p}<0.05$.

\section{Results}

Systemic administration of $\mathrm{MnCl}_{2}$ through i.p injections caused efficient uptake of $\mathrm{Mn}^{2+}$; our results show that $\mathrm{MnCl}_{2}$ administration provides visual enhancement of the 
different regions of the spinal cord. Analysis of MRI-T $T_{1}$ maps show significant changes post $\mathrm{MnCl}_{2}$ administration. These shorter $\mathrm{T}_{1}$ values serves as the basis for contrast enhancement and delineating specific anatomical structures in the spinal cord.

Figure 1 demonstrates $T_{1}$ weighted images of rat spinal cord before and after $\mathrm{MnCl}_{2}$ injection. Images are from L4 lumbar vertebrae level obtained with $69 \mu \mathrm{m}$ inplane resolution. $\mathrm{T}_{1}$ weighted images of spinal cord from another $\mathrm{Mn}^{2+}$ injected rat is shown in Figure 1B with the corresponding coronal anatomical section. Interestingly, the superficial layers, which consist mostly of the terminations of primary afferent nociceptive fibers and neurons of lamina I and substantia gelationsa can be visualized in the control MRI slice but are much more enhanced in $\mathrm{Mn}^{2+}$ enhanced MRI slice. In rats that received $\mathrm{Mn}^{2+}$, additional $\mathrm{T}_{1}$ enhancement can be seen in the ventro-lateral grey matter, which is a region that contains large motor neurons.

\section{Reduction of $\mathrm{T}_{1}$-Signals in $\mathrm{MnCl}_{2}$ injected rats show increased contrast}

\section{enhancement of grey matter}

MRI images of thoracic segments were obtained $48 \mathrm{~h}$ after $\mathrm{MnCl}_{2}$ injection. Signal enhancement in different spinal cord regions was calculated and normalized according to the nearby muscle. Signal enhancement within grey matter layers and proximate grey matter regions that contain small diameter interneuron populations in $\mathrm{Mn}^{2+}$ injected rats and control are demonstrated in Figure 2. Figure 2A shows three thoracic segments and anatomically defined ROIs. Laminar regions (I-III), (V-VII) and (VIII-IX) correspond to ROIs $(3,6,9) ;(2,5,8)$ and $(1,4,7)$ respectively. Figure 2 B show correlation fit curves for signal intensity values at six different repetition times for all measured ROIs. Figure 2C shows that the mean $\mathrm{T}_{1}$ values were significantly different 
before $(1.5627 \pm 0.01 \mathrm{~s})$ and after $\mathrm{MnCl}_{2}(1.4411 \pm 0.02 \mathrm{~s})$ administration $(\mathrm{p}=0.0007)$ in laminae I-III region. In Laminae V-VII region, there was a significant decrease in $\mathrm{T}_{1}$ (pre- $\mathrm{MnCl}_{2}: 1.5508 \pm 0.017 \mathrm{~s} ;$ post- $\left.\mathrm{MnCl}_{2}: 1.43511 \pm 0.025 \mathrm{~s}\right)(\mathrm{p}=0.02)$ as well. $\mathrm{T}_{1}$ values in VIII-IX region were $1.56 \pm 0.02 \mathrm{~s}$ before $\mathrm{MnCl}_{2}$ administration. Post $\mathrm{MnCl}_{2}, \mathrm{~T}_{1}$ values were reduced to $1.442 \pm 0.021 \mathrm{~s}, \mathrm{p}=0.0007$.

\section{Reduction of $T_{1}$ signal in corticospinal and spinalcortical white matter post}

\section{$\mathrm{MnCl}_{2}$ administration}

We sought to calculate the signal enhancement in white matter tracts post $\mathrm{MnCl}_{2}$, specifically in regions of ascending and descending tracts. Figure $\mathbf{3 A}$ shows a representative $\mathrm{MnCl}_{2}$ image with ROIs corresponding to spinalcortical (sensory pathway that conveys impulses concerning position of different body parts) and corticospinal (involved with voluntary motor function and sensory impulses) regions. Data from Figure 3B shows mean $T_{1}$ values for spinalcortical and corticospinal white matter regions before and post $\mathrm{MnCl}_{2}$ treatment. The changes in the mean $\mathrm{T}_{1}$ values for the spinalcortical region (Red ROI in middle) before $(1.5458 \pm 0.016 \mathrm{~s})$ and after $(1.4629 \pm$ $0.025 \mathrm{~s}) \mathrm{MnCl}_{2}$ were significant $(\mathrm{p}=0.003)$. The descending motor pathway (corticospinal) also had a significant visual enhancement (pre- $\mathrm{MnCl}_{2}, 1.5368 \pm 0.02 \mathrm{~s}$ ) vs (post- $\mathrm{MnCl}_{2}, 1.4467 \pm 0.02 \mathrm{~s}$ ), $\mathrm{p}=0.002$. Thus, our results show that MEMRI is effective in the contrast enhancement of regions that pertain to both sensory and motor pathways.

Next, we used histological methods to delineate specific regions of interest in the spinal cord slices obtained from the same rats following the imaging session. DAB staining of coronal $\mathrm{MnCl}_{2}$ administered $\mathrm{L} 4 \mathrm{~T}_{1}$ regions revealed areas of high cell density (Figure 4A). This was reflected as a hyper-intense MRI signal (area outlined in black). 
The second high intensity MRI region was identified as an area that has a large population of motor neurons (area outlined in red). Both histology and corresponding MRI images are placed side by side for comparison. Figure 4B further delineated in detail the regions identified in Figure 4A. The boxed region labelled A was identified as laminae regions I \& II, which contain a high density of cells that responds to noxious and thermal stimuli (18). Figure 4A also shows laminae IX region with the presence of large neurons. These have previously been identified as somatic motor neurons that innervate muscles (19). Figure 4C shows effective labelling of the spinal cord with mature neuronal marker $(\mathrm{NeuN})$ and nuclear staining (DAPI). Extensive labelling with NeuN was seen in both dorsal and ventral grey matter of the spinal cord. Double labelling with NeuN and DAPI were visualized and reported.

\section{Discussion}

The use of MEMRI for in vivo neuronal tracing has been on the rise. Here, we showed that MEMRI can visually enhance specific regions of the spinal cord through decreased $\mathrm{T}_{1}$ changes post $\mathrm{MnCl}_{2}$ administration. Our results demonstrate efficient $\mathrm{Mn}^{2+}$ transport and accumulation in corticospinal and spinalcortical pathways after i.p. injection.

Different spinal cord regions are associated with various neurological disorders such as ALS, multiple sclerosis, nerve damage and chronic pain. There is a growing interest in using cellular and stem cell therapies to combat different motor neurons disorders (20). Currently, the only approaches to assessing the efficacy of these strategies has either been behavioral testing or histology with ex vivo imaging methods. The possibility of imaging in vivo and non-invasively the function of different cell population 
within the spinal cord grey matter, opens a new frontier in the assessment of different therapeutic strategies.

Pathologies of the spinal cord tracts have been detected using diffusion tensor and functional magnetic imaging techniques $(21,22)$ and MEMRI $(15,16)$. Recent reports have focused on using MEMRI to assess neuronal damage post spinal cord injury (23) and in assessing signal transduction mechanisms in the spinal cord (24). These reports demonstrate the applicability of the techniques to follow gross architectural and to some degree, functional changes of the spinal cord.

It has been shown that $\mathrm{Mn}^{2+}$ ion enter cells through voltage gated calcium channels $(10,25)$. Different classes of these channels are used to amplify and increase the output of the cells. At the lumber 4 level of the spinal cord, the motor neurons in lamina IX are larger in size compared to other neurons in the spinal cord grey matter at that level. Since their surface area is large (more than $500,000 \mu \mathrm{m}^{2}$ ) they exhibit more $\mathrm{Ca}^{2+}$ channels per cell and therefore the accumulation of $\mathrm{Mn}^{2+}$ within them is greater. In contrast, lamina I is composed of small and medium sized cells that respond specifically to noxious stimuli, and lamina II of the spinal cord is composed of tightly packed small cells that are also involved in pain processing. However, dense cell architecture in these laminae may underlie the significant enhancement in the signal of that region. Interestingly, there is an enormous interest both in visualizing cells function in the upper dorsal horn laminae for drug development that targets acute and chronic pain, as well as in the large motorneurons in lamina IX in respect to following therapeutic strategies for motorneurons disorders. 
Systemic manganese infusion was applied previously to study cerebral architecture in normal (26) and specific mutation in rodents $(27,28)$. Indeed, a difference in the cerebral architectural of the mutant mice was visualized using this method. We show that MEMRI is successful in diffrentiating different grey matter regions within the spinal cord and can be used for longitudinal studies of spinal cord physiology in vivo.

\section{Author contributions}

VK, JX, GAM, AK, SA and GP designed experiments, acquired and analyzed data. VK and GP wrote and the edited the paper.

\section{Declaration of Competing Interest}

There is no conflict of interest for any of the authors.

\section{Acknowledgements}

This work was supported by National Institute of Health/ National Institute of Neurological Disorders and Stroke R01NS098231 and NIH/NINDS R01NS072171 grants. The authors thank the Intramural National Institute of Neurological Disorders and Stroke and the National Heart, Lung, and Blood Institute for support

\section{Figure legends}

Figure 1. (A) $\mathrm{T}_{1}$ weighted images of coronal $\mathrm{L} 4$ spinal level in control and $\mathrm{Mn}^{2+}$ injected rats acquired 48 hours following $\mathrm{Mn}^{2+}$ i.p. administration showing increased contrast enhancement. (B) Shows half of the cord from a $\mathrm{Mn}^{2+}$ injected rat labelled with the corresponding laminae anatomical sections. 
Figure 2. (A) Specific laminae regions across the thoracic section of the rat spine showing regions of interest (ROI) 1-9 (B) Individual representation of $\mathrm{T}_{1}$ values across ROI regions 1-9 before (black) and after (red) $\mathrm{MnCl}_{2}$ administration, with post- $\mathrm{MnCl}_{2}$ groups showing lower $\mathrm{T}_{1}$ values $(\mathbf{C})$ Grouped pre- $\mathrm{MnCl}_{2}$ laminae $\mathrm{T}_{1}$ average compared to post- $\mathrm{MnCl}_{2}$ laminae average. Paired $\mathrm{T}$-Test analysis of laminae were significantly different $(\mathrm{p}<0.05)$.

Figure 3. (A) Specific regions (red ROI) of the spinal cord that correlate to ascending and descending tracts. (B) Grouped pre- $\mathrm{MnCl}_{2}$ white matter averaged areas compared to post-MnCl2 averaged white matter areas. Paired T-Test analysis of white matter tracts, pre and post, were significantly different $(\mathrm{p}<0.05)$.

Figure 4. (A) Coronal L4 $\mathrm{T}_{1}$ weighted image with the corresponding histology taken from a $\mathrm{Mn}^{2+}$ injected rat. Note that the hyper-intense MRI regions match regions where high cell density (black) and large motor neurons are found (red). (B) A coronal L4 histology image. Laminae I \& II have high density cell bodies (box labelled A) and respond strongly to noxious and thermal stimuli. Lamina IX (box labelled B) consists of several distinct clusters of large somatic motor neurons that innervate muscles in the lumber level. Boxed sections A and B has been enlarged to show hyper intense cell bodies and motor neurons. (C) Coronal sections showing neuronal labelling (NeuN in green), nuclei labelling (DAPI in green) and merged image of NeuN and DAPI.

\section{References}

1. Rexed B (1954) A cytoarchitectonic atlas of the spinal coed in the cat. Journal of comparative neurology 100(2):297-379.

2. Mu X, He J, Anderson DW, Springer JE, \& Trojanowski JQ (1996) Altered expression of bcl $\square 2$ and bax mRNA in amyotrophic lateral sclerosis spinal cord 
motor neurons. Annals of Neurology: Official Journal of the American Neurological Association and the Child Neurology Society 40(3):379-386.

3. $\quad \mathrm{Mu}$ X, Springer JE, \& Bowser R (1997) FAC1 expression and localization in motor neurons of developing, adult, and amyotrophic lateral sclerosis spinal cord. Experimental neurology 146(1):17-24.

4. $\quad$ Pearn J (1980) Classification of spinal muscular atrophies. The Lancet 315(8174):919-922.

5. Cross DJ, et al. (2006) In vivo imaging of functional disruption, recovery and alteration in rat olfactory circuitry after lesion. Neuroimage 32(3):1265-1272.

6. Pelled G, Bergman H, Ben-Hur T, \& Goelman G (2007) Manganese-enhanced MRI in a rat model of Parkinson's disease. Journal of Magnetic Resonance Imaging 26(4):863-870.

7. Van Der Linden A, et al. (2002) In vivo manganese-enhanced magnetic resonance imaging reveals connections and functional properties of the songbird vocal control system. Neuroscience 112(2):467.

8. Saar G \& Koretsky AP (2018) Manganese Enhanced MRI for Use in Studying Neurodegenerative Diseases. Frontiers in neural circuits 12.

9. Duong TQ, Silva AC, Lee SP, \& Kim SG (2000) Functional MRI of calciumdependent synaptic activity: cross correlation with CBF and BOLD measurements. Magn Reson Med 43(3):383.

10. Lin YJ \& Koretsky AP (1997) Manganese ion enhances T1 $\square$ weighted MRI during brain activation: an approach to direct imaging of brain function. Magnetic resonance in medicine 38(3):378-388.

11. Yu X, Wadghiri YZ, Sanes DH, \& Turnbull DH (2005) In vivo auditory brain mapping in mice with Mn-enhanced MRI. Nat Neurosci 8(7):961-968.

12. Aoki I, Wu Y-JL, Silva AC, Lynch RM, \& Koretsky AP (2004) In vivo detection of neuroarchitecture in the rodent brain using manganese-enhanced MRI. Neuroimage 22(3):1046-1059.

13. Saar G, et al. (2018) Anatomy, functionality, and neuronal connectivity with manganese radiotracers for positron emission tomography. Molecular Imaging and Biology 20(4):562-574.

14. Sudarshana D, et al. (2019) Manganese-Enhanced MRI of the Brain in Healthy Volunteers. American Journal of Neuroradiology 40(8):1309-1316.

15. Bilgen $M$ (2006) Imaging corticospinal tract connectivity in injured rat spinal cord using manganese-enhanced MRI. BMC Med Imaging 6:15.

16. Stieltjes B, et al. (2006) Manganese-enhanced magnetic resonance imaging for in vivo assessment of damage and functional improvement following spinal cord injury in mice. Magn Reson Med 55(5):1124-1131.

17. Martirosyan NL, Bennett KM, Theodore N, \& Preul MC (2010) Manganeseenhanced magnetic resonance imaging in experimental spinal cord injury: correlation between T1-weighted changes and Mn2+ concentrations. Neurosurgery 66(1):131-136.

18. Zeilhofer HU, Wildner H, \& Yévenes GE (2012) Fast synaptic inhibition in spinal sensory processing and pain control. Physiological reviews 92(1):193-235.

19. Watson C, Paxinos G, \& Kayalioglu G (2009) The spinal cord: a Christopher and Dana Reeve Foundation text and atlas (Academic press). 
20. Hedlund E, Hefferan MP, Marsala M, \& Isacson O (2007) Cell therapy and stem cells in animal models of motor neuron disorders. Eur J Neurosci 26(7):17211737.

21. Kim JH, et al. (2007) Noninvasive diffusion tensor imaging of evolving white matter pathology in a mouse model of acute spinal cord injury. Magn Reson Med 58(2):253-260.

22. Krishnan VS, et al. (2019) Multimodal evaluation of TMS-induced somatosensory plasticity and behavioral recovery in rats with contusion spinal cord injury. Frontiers in neuroscience 13:387.

23. Freitag MT, et al. (2015) Monitoring of Short $\square$ Term Erythropoietin Therapy in Rats with Acute Spinal Cord Injury Using Manganese $\square$ Enhanced Magnetic Resonance Imaging. Journal of Neuroimaging 25(4):582-589.

24. Cha M, Lee K, Won JS, \& Lee BH (2019) Manganese-enhanced magnetic resonance imaging of the spinal cord in rats with formalin-induced pain. Neuroscience research.

25. Simpson P, Challiss R, \& Nahorski S (1995) Divalent cation entry in cultured rat cerebellar granule cells measured using Mn2+ quench of fura 2 fluorescence. European Journal of Neuroscience 7(5):831-840.

26. Silva AC, et al. (2008) Detection of cortical laminar architecture using manganese-enhanced MRI. J Neurosci Methods 167(2):246-257.

27. Angenstein F, et al. (2007) Manganese-enhanced MRI reveals structural and functional changes in the cortex of Bassoon mutant mice. Cereb Cortex 17(1):2836.

28. Wadghiri YZ, et al. (2004) Manganese-enhanced magnetic resonance imaging (MEMRI) of mouse brain development. NMR Biomed 17(8):613-619. 
bioRxiv preprint doi: https://doi.org/10.1101/781823; this version posted September 25, 2019. The copyright holder for this preprint (which was not certified by peer review) is the author/funder, who has granted bioRxiv a license to display the preprint in perpetuity. It is made available under aCC-BY-NC-ND 4.0 International license. 


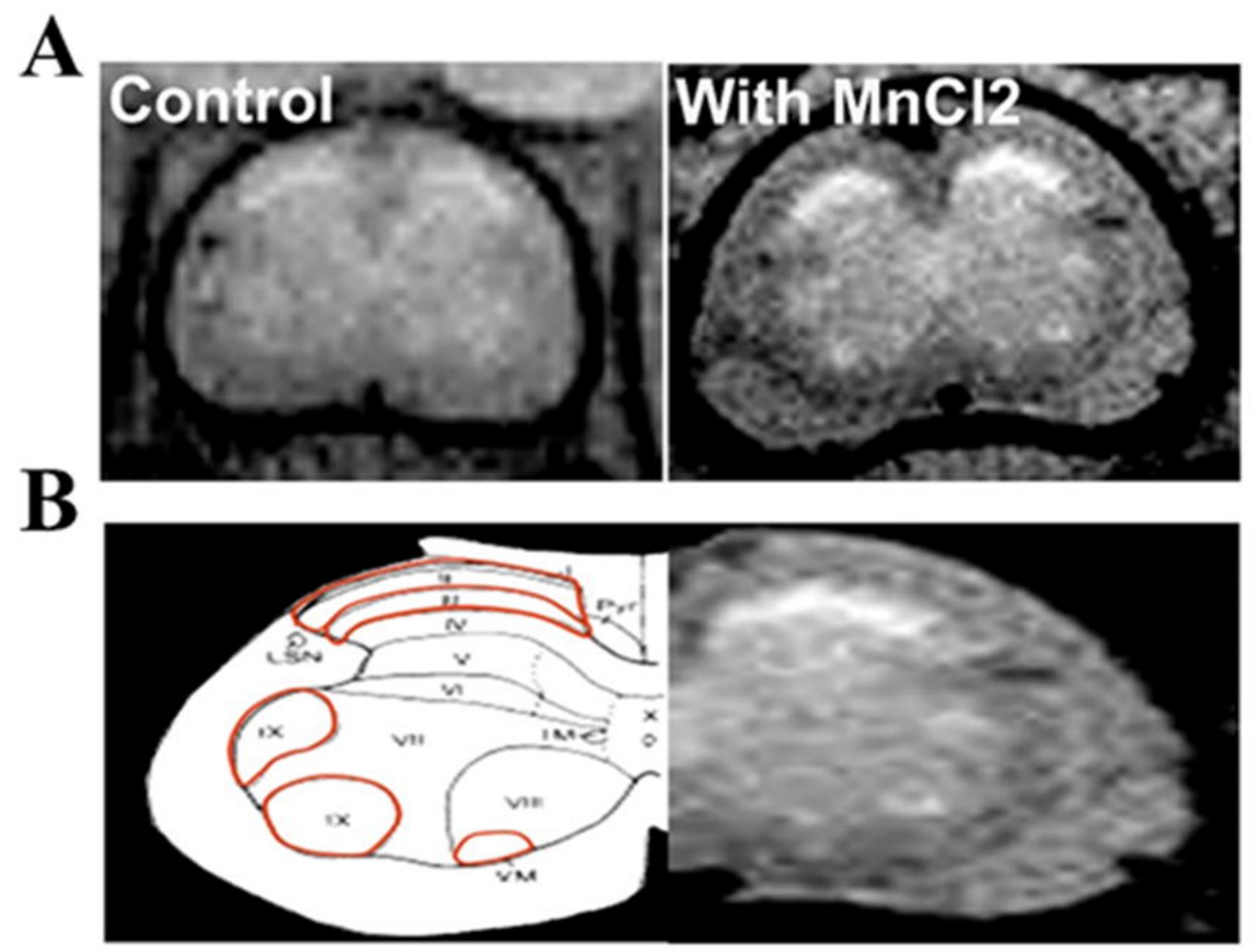


A

B

$=$ Before $\mathrm{Mn}=$ After $\mathrm{Mn}$
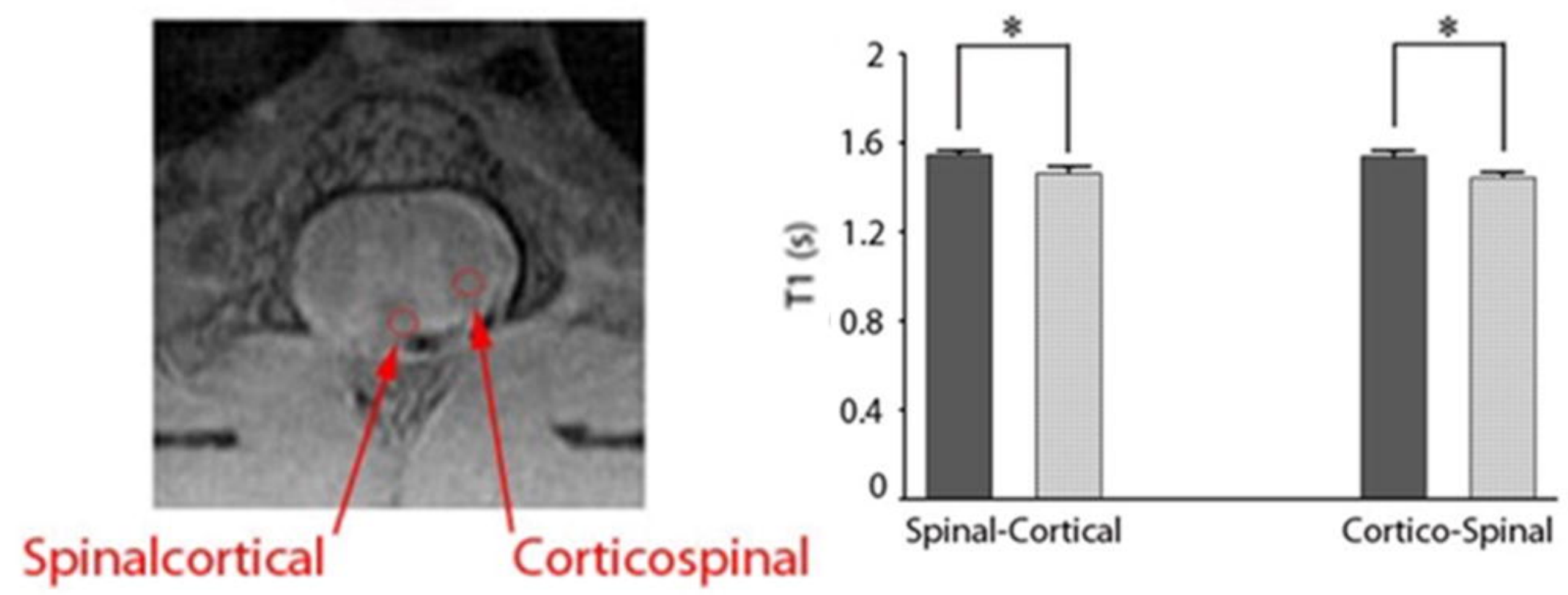


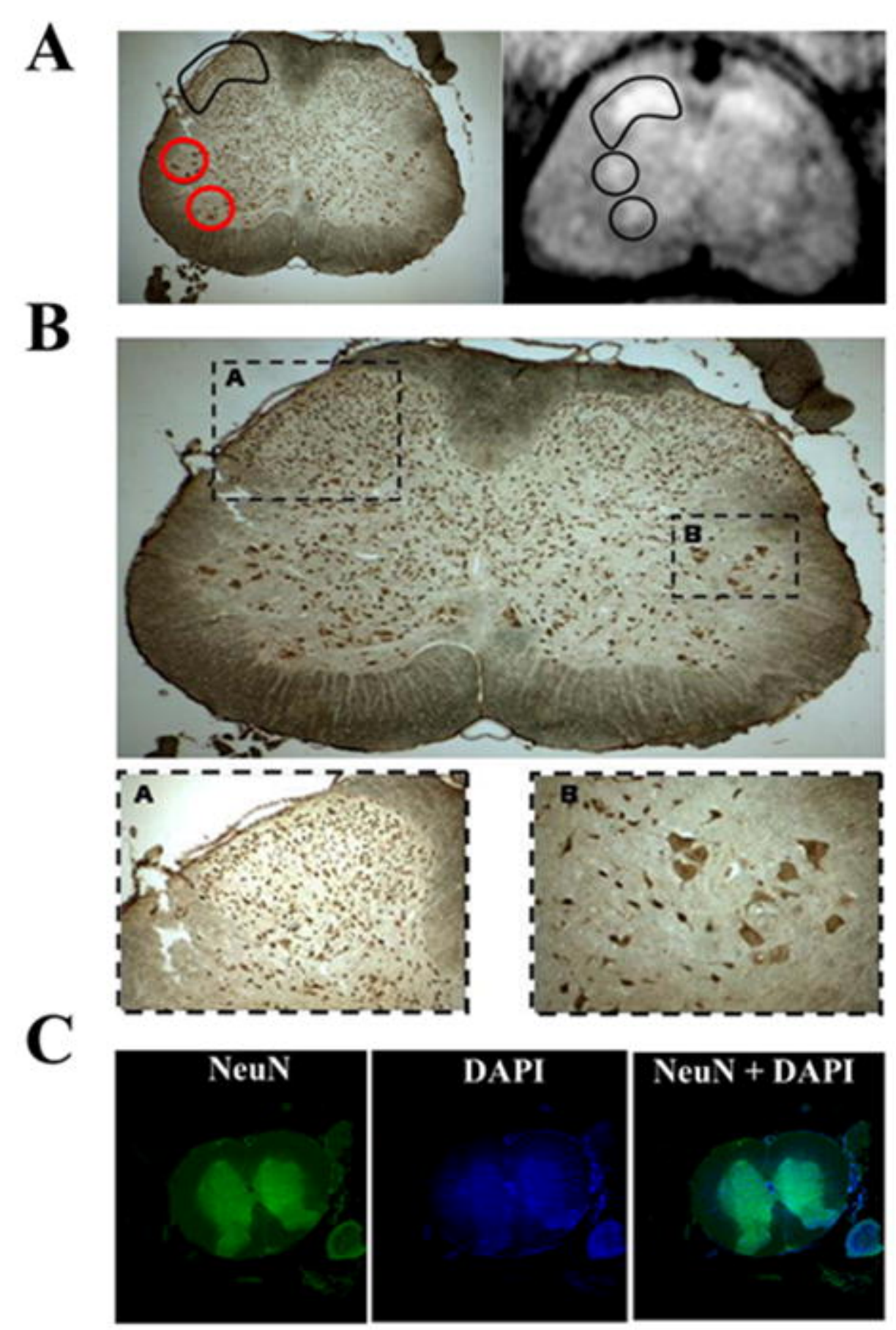

